\title{
Novel method for patterned fabric inspection using Bollinger bands
}

\author{
Henry Y. T. Ngan \\ University of Hong Kong \\ Industrial Automation Research Laboratory \\ Department of Electrical and Electronic \\ Engineering \\ Pokfulam Road, Hong Kong
}

\author{
Grantham K. H. Pang \\ University of Hong Kong \\ Industrial Automation Research Laboratory \\ Department of Electrical and Electronic \\ Engineering \\ Pokfulam Road, Hong Kong \\ E-mail: gpang@eee.hku.hk
}

\begin{abstract}
This paper introduces a new application of Bollinger bands for defect detection of patterned fabric. A literature review on previous designed methods for patterned fabric defect detection will be depicted. For data analysis, Bollinger bands are calculated based on standard deviation and are originally used in the financial market as an oversold or overbought indicator for stock. The Bollinger bands method is an efficient, fast and shift-invariant approach, that can segment out the defective regions on the patterned fabric with clear and crystal clean images. The new approach is immune of the alignment problem that often happens in previous methods. In this paper, the upper band and lower band of Bollinger bands, which are sensitive to any subtle change in the input data, have been developed for use to indicate the defective areas in patterned fabric. The number of standard deviation and length of time of Bollinger bands can be easily determined to obtain excellent detection results. The proposed method has been evaluated on three different patterned fabrics. In total, 165 defect-free and 171 defective images have been used in the evaluation, where $98.59 \%$ accuracy on inspection has been achieved. () 2006 Society of Photo-Optical Instrumentation Engineers. [DOI: 10.1117/1.2345189]
\end{abstract}

Subject terms: Bollinger bands; patterned fabric inspection; defect detection; texture analysis.

Paper 050551R received Jul. 6, 2005; revised manuscript received Oct. 3, 2005; accepted for publication Jan. 13, 2006; published online Sep. 6, 2006.

\section{Introduction}

Nowadays, most inspections on weaving fabric are visually conducted by workers. Repetitive work for inspection, recording, and correction are needed every day and a large labor force is involved for visual inspection. With the limitations of human eyes, there are frequent mistakes during such continuous work. From the point of view of costeffectiveness, automated fabric inspection can reduce the labor cost and increase the accuracy of inspection. Automated fabric inspection machines have been developed but they can only tackle the "unpatterned" fabric such as plain and twill fabrics. ${ }^{1-3}$ There is no designed pattern on the fabric surface. Many methods such as autoregressive method, ${ }^{4}$ neural network, ${ }^{5-9}$ Fourier transform, ${ }^{10-13}$ Gabor filters, ${ }^{14-18}$ wavelet transform, ${ }^{19-26}$ structural method, ${ }^{27}$ and some spatial and spectral approaches ${ }^{28}$ have been developed for them. Most detection success rates of these methods can reach over $95 \%$. In fact, as Puig ${ }^{29}$ mentioned, no specific method has proved to be superior in texture detection. The previous methods above were implemented mainly on unpatterned fabric like plain and twill fabric. However, only a few researchers have focused on patterned fabric inspection. There is much room for researchers to explore different approaches on dealing with patterned fabric. Our research aims to segment out the defects from patterned fabric background and achieve a higher accuracy of defect detection on patterned fabric.

The word "patterned" has a special meaning in fabric.
The patterned fabric is defined as fabric with repetitive basic units in its design. Under the class of patterned fabric, there are still many categories. The pattern unit can range from the simplest charter boxes (repetitive pattern) and dots, to the most complicated multiple flowers, animals, or designed patterns (complex pattern). Figure 1 briefly illustrates on the classification of fabrics.

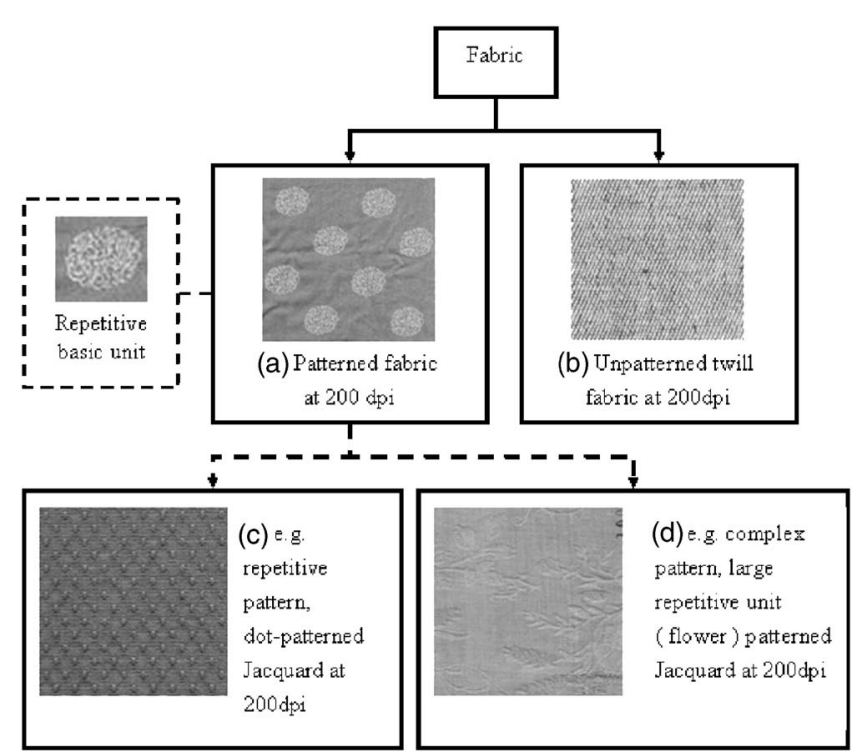

Fig. 1 Classification of fabric. 


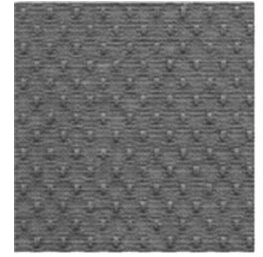

a.

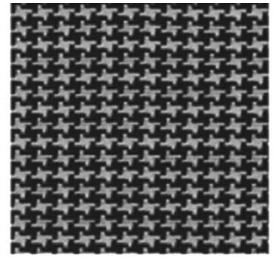

b.

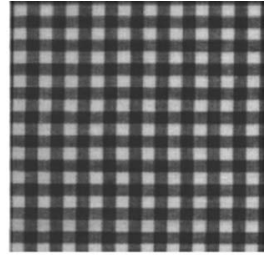

c
Fig. 2 Three patterned fabrics used in this paper: (a) dot-patterned, (b) star-patterned, and (c) box-patterned.

This paper describes an effective and efficient method to detect defects on patterned fabric. Because this is a relatively new topic and there are lots of patterned fabrics on the market, this research has focused on some simpler patterns. To illustrate the usefulness of our developed method, three different repetitive patterned fabric materials, the dotpatterned, star-patterned, and box-patterned fabrics shown in Fig. 2 have been used in the evaluation. A literature review of prior patterned fabric inspection methods is given in Sec. 2. Next, Bollinger bands (BB) for defect detection are introduced in Sec. 3. The background and the interpretation of BB applied to images are described. Then, detailed evaluation based on the dot-patterned fabric is given in Sec. 4. This is followed by more evaluation results based on star-patterned and box-patterned fabrics in Sec. 5. A summary with conclusions is delivered in Sec. 6.

\section{Literature Review of Defect Detection Methods on Patterned Fabrics}

Most researchers have only focused on defect detection of unpatterned fabric. The traditional methods for unpatterned fabric inspection cannot be used on patterned fabric inspection. The yarn structures of patterned fabric are more complex and much finer than unpatterned fabric so that deformation or distortion in patterns [Fig. 1(a)] are often found during the acquisition of images. Also, a certain amount of shift in patterned fabric always happens in the input acquired image. All these would highly increase the difficulties on feature extraction and affect the feasibility to apply the traditional methods such as the co-occurrence matrix approach. Therefore, in the literature survey, only a few research studies are found to be related to patterned fabric inspection.

$\mathrm{Li}^{30}$ and Sandy ${ }^{31}$ have used a subtraction method, which was previously applied in printed circuit boards (PCBs) by

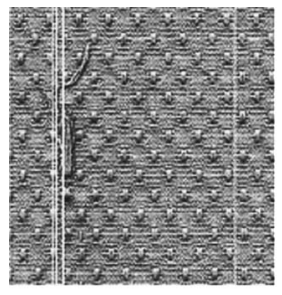

a. Netting Multiple

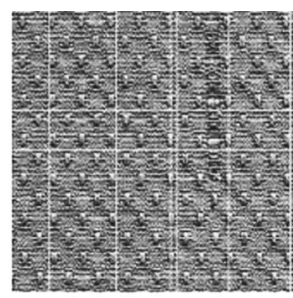

b. Broken End
Fig. 3 Two typical defective patterned images examples of misclassification with threshold signatures of multiplication hash function presented on defective patterned images (detected on horizontal and vertical sides).

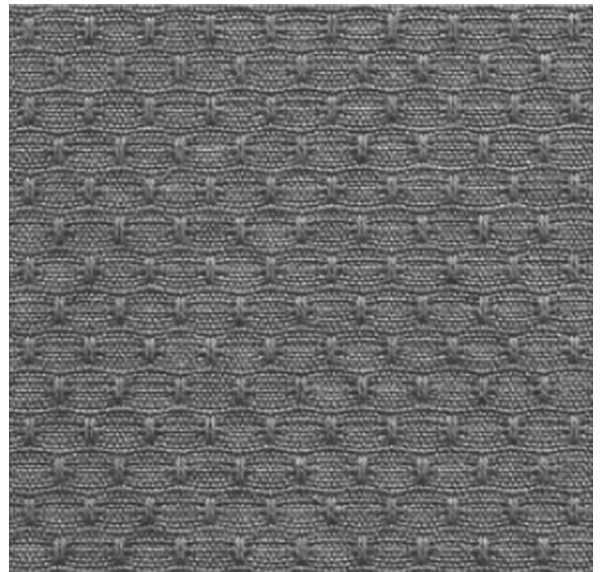

Fig. 4 Dot-patterned jacquard fabric.

Chin and Harlow, ${ }^{32}$ for defect detection on lace, which is one kind of patterned fabric with a fine and sophisticated pattern of threads. This subtraction method is equivalent to an EXCLUSIVE-OR operation. In detection, the testing lace sample was subtracted by a prototype version of the same pattern while the repeat distance of the pattern on the testing lace sample was found by autocorrelation. Thresholding was then used and a neural network is used to detect and classify the different kinds of defects. This subtraction method was classified as traditional, which is not the same as the golden image subtraction method mentioned below. The main weakness of this traditional subtraction method is the alignment problem between the reference image and test image. Costa et al. ${ }^{33}$ also admitted that pattern matching is the most difficult part for the traditional image subtraction method on patterned fabric inspection. Thomas ${ }^{34}$ proposed to use the image block densitometric profile to analyze the mean value of every row or column in the test image for defect detection. This profile analysis method was evaluated with simulated images and Thomas declared the method could apply on the simple pattern only.

A similar idea using a hash function emerged from Baykal et al. ${ }^{35,36}$ on patterned texture because the checksum hash function of Baykal is consistent with the image densitometric profile. Baykal developed four types of hash

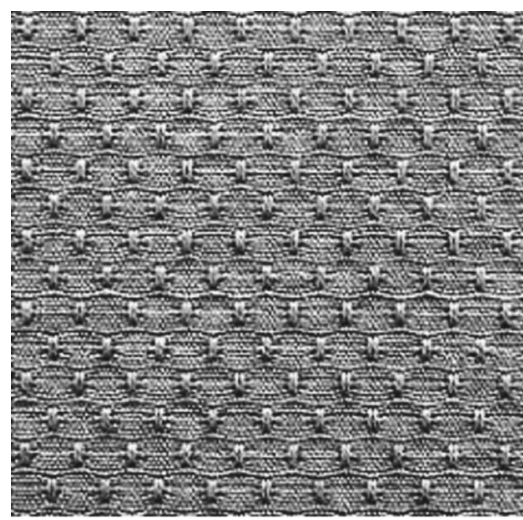

a

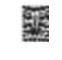

b
Fig. 5 (a) After histogram equalization. (b) A repetitive unit. 


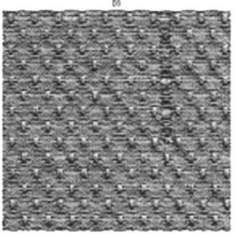

a. B roken End

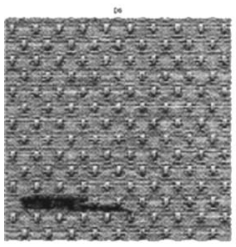

e. Thin Bar

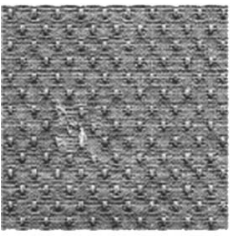

i. Loose Pick

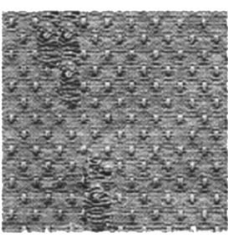

b. Holes

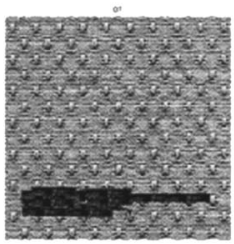

f. Thick Bar

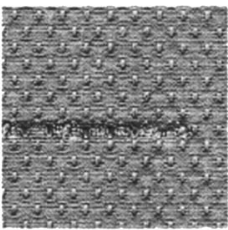

j. Missing Pick

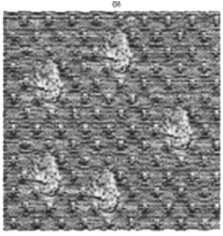

c. Knots

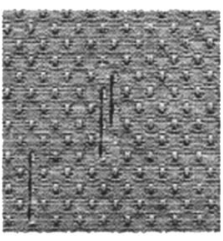

g. Oil Warp

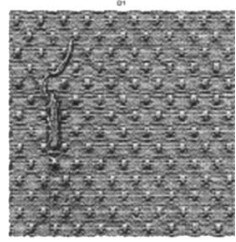

d. Netting Multiple

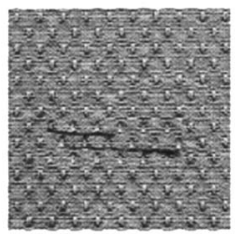

h. Oil Weft

Fig. 6 Six types of defective histogram equalized images with (a) broken end, (b) holes, (c) knots, (d) netting multiple, (e) thin bar, (f) thick bar, (g) oil warp, (h) oil weft, (i) loose pick, and (j) missing pick.

functions and used them as a preprocessing step to enhance the defects. The technique of the hash function is from cryptography, which is used for encryption and decryption. Any little changes that happened in data transmission will be revealed by the hash function. In a preliminary investigation of the Baykal method for patterned fabric defect detection, the method seems too sensitive to small distortion inherent in the pattern. Hence, the method may give false alarms and is not effective. Figure 3 shows some results when threshold is applied on the dot-patterned texture.

Bennamoun and Bodnarova ${ }^{37}$ applied six standard techniques on unpatterned fabric defect detection and tried to apply an optimized Gobar filter on jacquard fabric inspection. However, only two different jacquard samples, with holes and smash flaws, respectively, were investigated under their Gabor filtering techniques. The detection results in

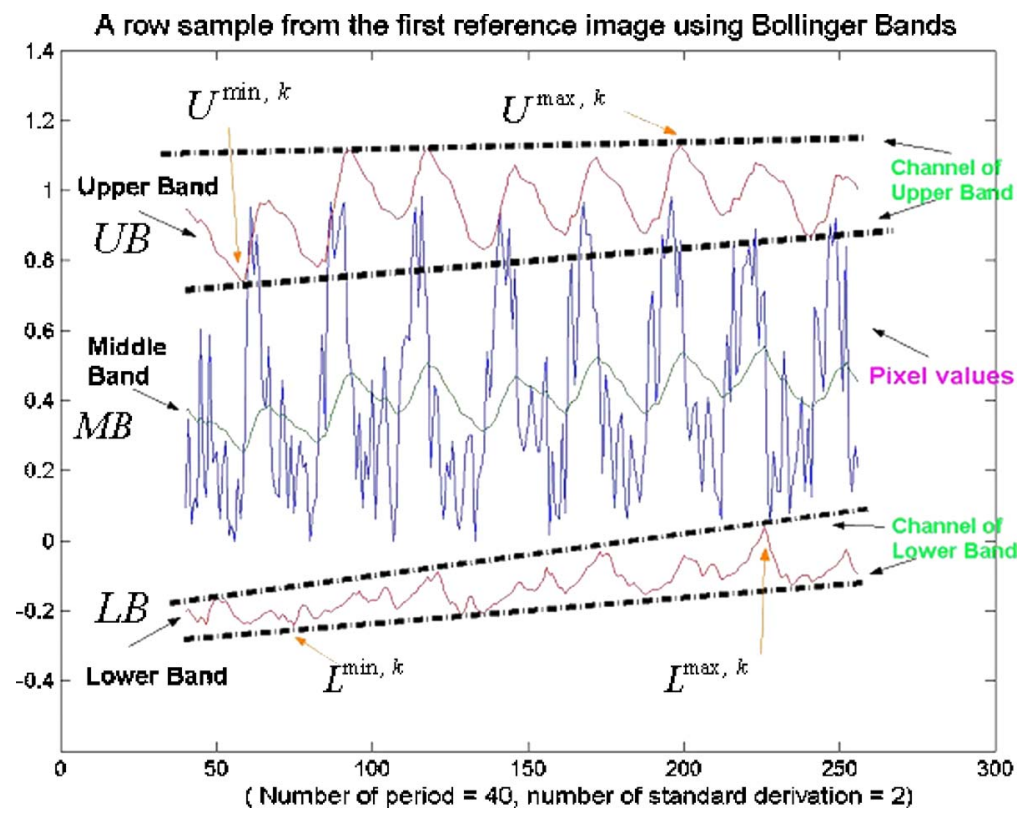

Fig. 7 Upper, middle, and lower BBs for a sample row from a reference image $(n=40, d=2)$. The upper limit of the upper band is labeled as $U^{\text {max }, k}$ and the lower limit of that band is labeled as $U^{\text {min, }} k$. The upper limit of lower band is labeled as $L^{\max , k}$ and the lower limit of that band is labeled as $L^{\min , k}$. 


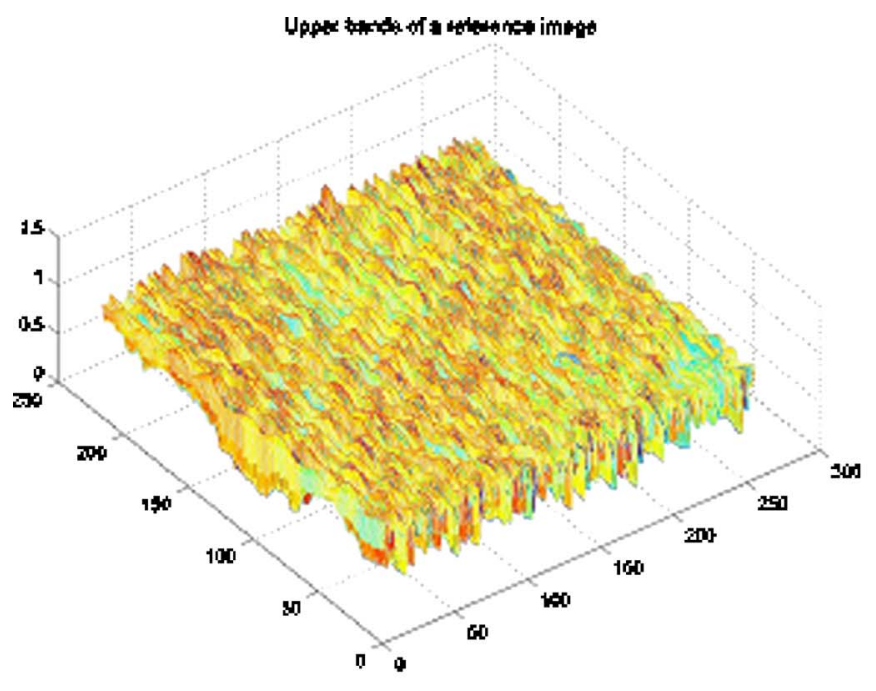

a

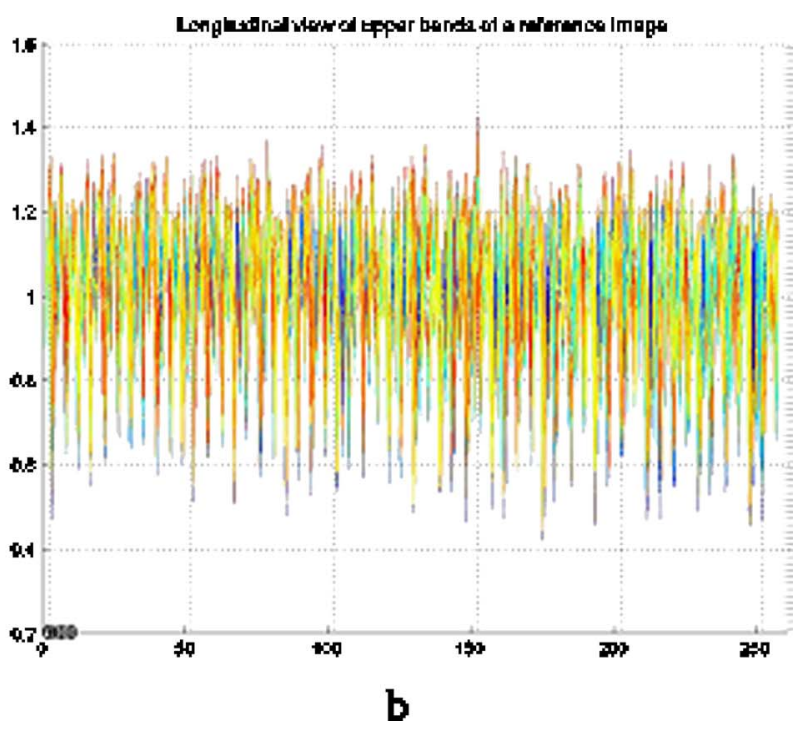

Fig. 8 Mesh diagrams of (a) overhead view, (b) longitudinal view for upper bands, on rows, of a reference image $(N 1=40, d 1=2)$.

that paper showed parts of the defective regions on original images, and there is no strong evidence that the Gabor filter will be effective enough on other kinds of defects existing in those two kinds of patterned jacquard fabric. Farooq et al. ${ }^{38}$ developed a mechatronic methodology to detect lace defects using a visual feedback approach. They claimed the system could adjust image rotation, scale, and translation parameters in a feedback control mode. After a carefully done alignment process, both the reference image and the test image would undergo a subtraction. Yet only three lace samples were tested under their proposed method. Neither of Bennamoun's and Farooq's methods on patterned fabric inspection have been widely evaluated with enough samples.
Ngan and Pang ${ }^{39}$ have developed a method of patterned fabric defect detection called the golden image subtraction (GIS) method, which is different from Sandy's subtraction method. The GIS method makes use of a golden image, which is larger than a repetitive unit, to carry out subtractions from the first pixel of the first row to the end of the last row for a test image. The golden image is similar to a convolution filter on the test image but performs the calculation of absolute mean of every subtraction in order to generate a GIS energy value. Then, the resultant matrix, with all energies from subtractions, will show periodic waveforms from a reference patterned sample. The defect region of a defective image will give a subtle change in the resultant matrix. However, not all types of defects can be

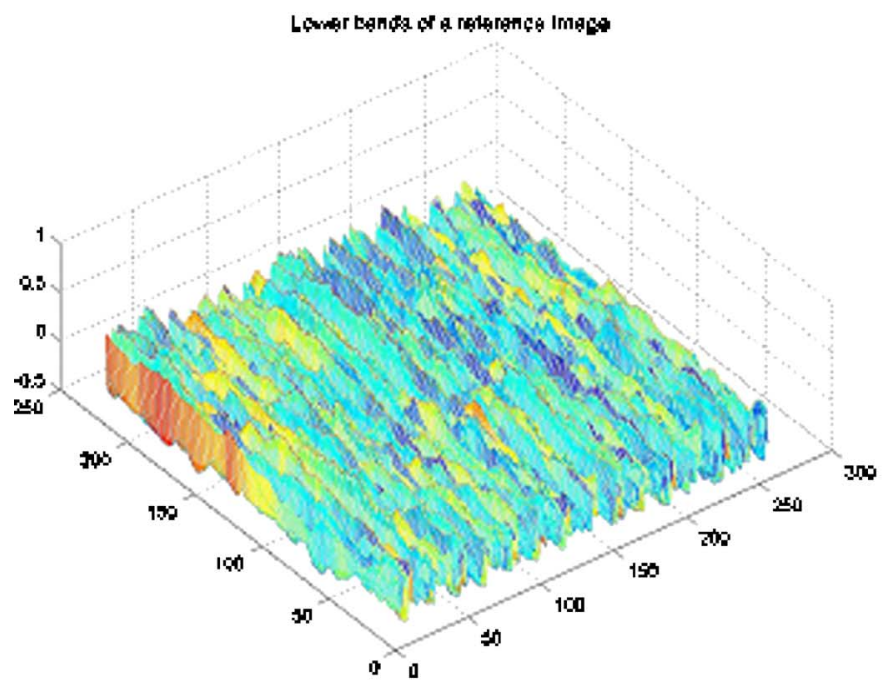

a

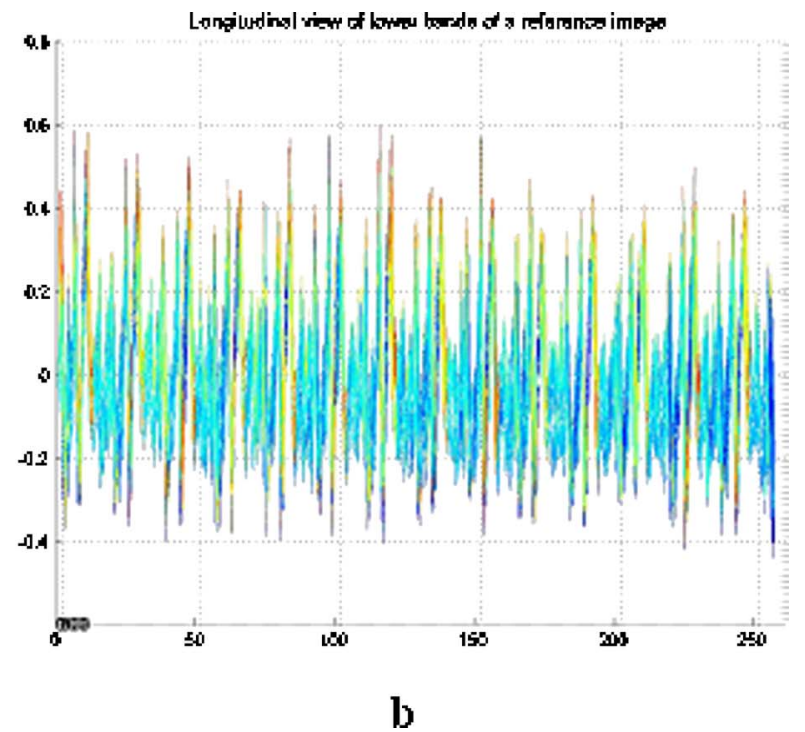

Fig. 9 Mesh diagrams of (a) overhead view, (b) longitudinal view for lower bands, on rows, of a reference image $(N 1=40, d 1=2)$. 

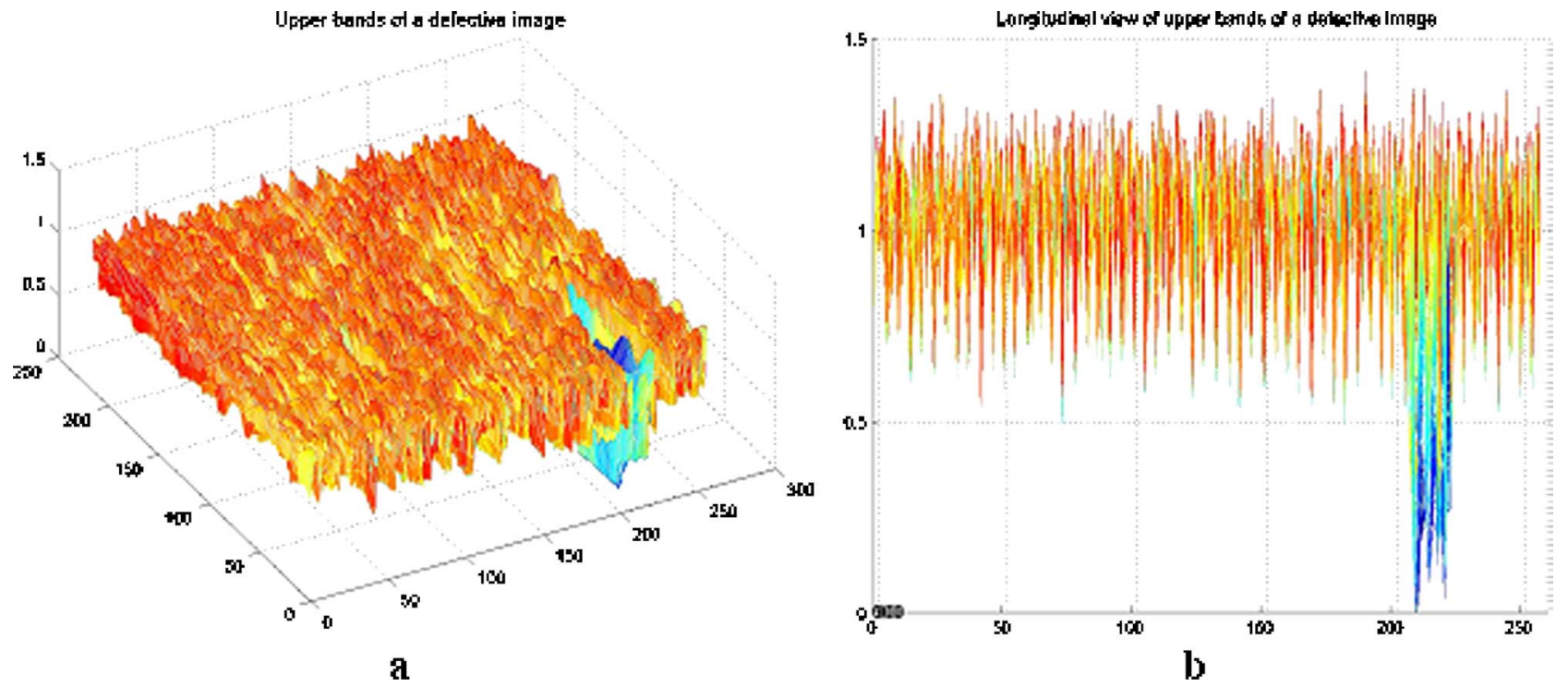

Fig. 10 Mesh diagrams of (a) overhead view, (b) longitudinal view for upper bands, on rows, of a defective image with thin bar, same as Fig. $6(\mathrm{e})(N 1=40, d 1=2)$.

successfully detected by the GIS method. The method is most effective for holes and thick bars on patterned fabric and an improved version of the GIS method has been developed with the use of wavelets for preprocessing.

Anagnostopoulos et al. ${ }^{40}$ suggested using a sliding window algorithm based on the standard deviation ratio between two concentric windows where one window is bounded by the other inside another window during sliding on the whole input image. Then, with the defect-free images, the standard derivation difference of near blocks should be quite smooth. This sliding window concept is quite similar to the GIS method. ${ }^{39}$ Anagnostopoulos et al. have applied their algorithm on unpatterned woven plain fabric only, whereas our method has been developed for patterned fabric inspection.

Ngan et al. ${ }^{41}$ have also developed a wavelet-based method for patterned fabric defect detection, and it has been evaluated on an image database of sixty patterned fabric samples. The wavelet transformed detailed subimages are used for defect detection and the results have been used to compare with the wavelet preprocessed GIS scheme. Yet small defects like small holes and loose pick

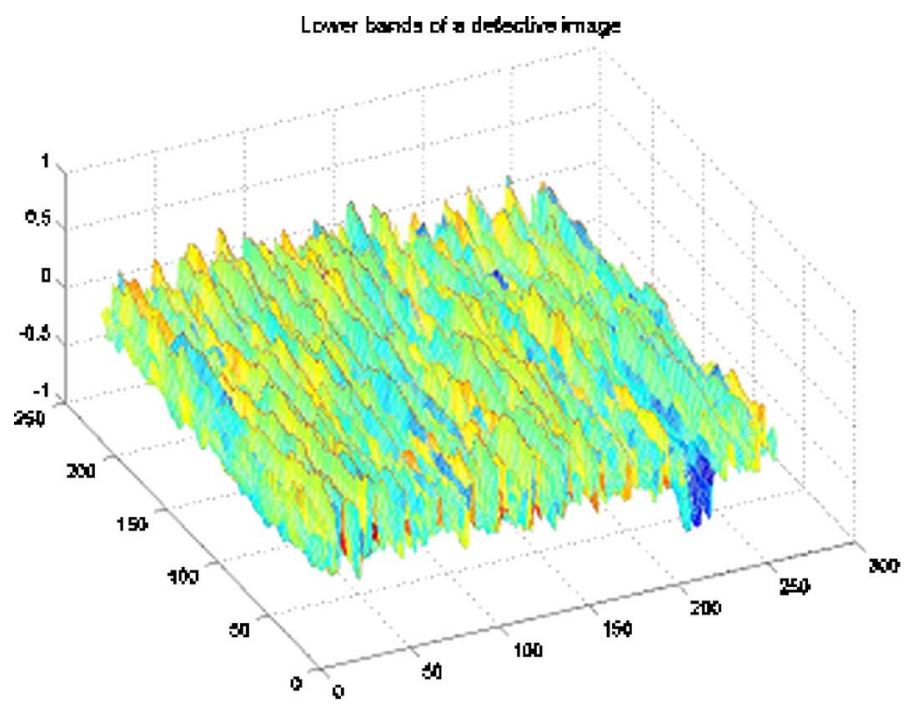

a

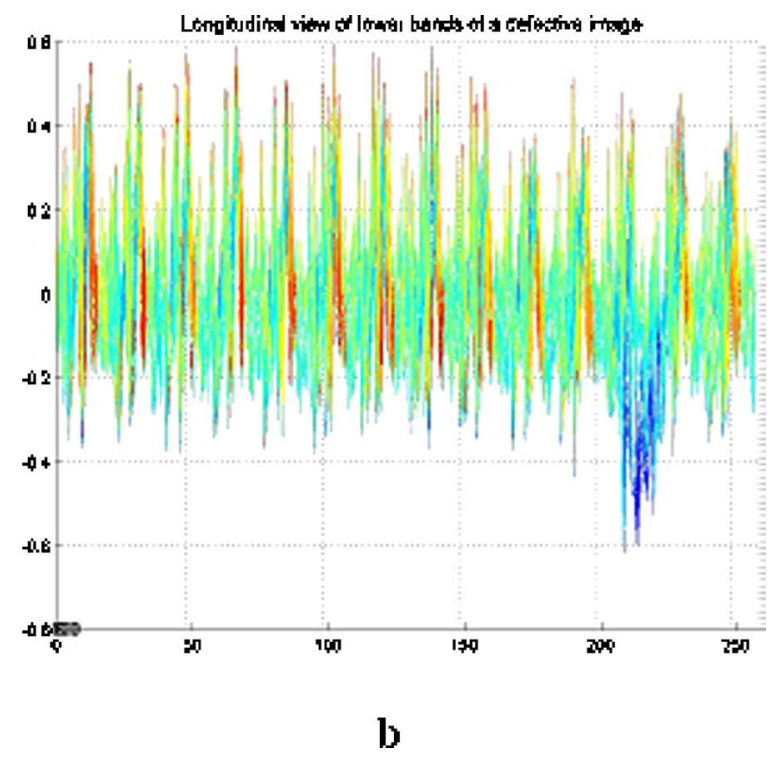

Fig. 11 Mesh diagrams of (a) overhead view, (b) longitudinal view for lower bands, on rows, of a defective image with thin bar, same as Fig. $6(\mathrm{e}) .(N 1=40, d 1=2)$. 
have been difficult to detect. The BB method developed in this paper has been shown to outperform the previous methods for patterned fabric inspection.

This paper presents a new application of $\mathrm{BB}$, and the method will first be evaluated with a dot-patterned designed fabric, which is shown in Fig. 4. A total of 230 images have been used. Histogram equalization has been performed on all images (e.g., Fig. 5) because the intensities of patterned fabric images may not be even enough for later processes in defect detection. Ten kinds of defects (broken end, holes, knots, netting multiple, thick bar, thin bar, oil warp, oil weft, loose pick and missing pick) as shown in Fig. 6 have been used for the evaluation. So, an enhancement of contrast for an even histogram can be achieved by the use of histogram equalization. All images for reference and testing have $256 \times 256$ pixels in gray level scale. The BB method in this paper is mainly for the gray scale images. MATLAB (version 6.5) has been used to implement the developed algorithm and to perform the evaluation. In Sec. 5, two more patterned fabrics, star-patterned and box-patterned, will be used for an extended evaluation.

\section{BB Method for Patterned Fabric Defect Detection}

BBs were designed by John Bollinger ${ }^{42}$ in 1983 and have been used for analyzing the trends in the stock markets. BB consist of three bands: upper, middle, and lower. BBs, especially for upper and lower bands, can enhance the pattern of a trend for a certain period. In other words, showing periodicity is the strength of this tool. In this method, patterned fabric can be considered as comprising many rows (columns), with a patterned design on each row (column). The principle of the BB method is that the patterned rows (columns) will generate periodic upper and lower bands. Any defective region in patterned fabric means that there would be a break of periodicity in the pattern. By the definition of BBs, these little abnormal changes in that region would lead to a prompt and huge variation in the standard deviation. Therefore, there should be a remarkable change in the upper band or lower band showing that significance. Of course, a good detection result can be obtained with the use of an appropriate bandwidth and period length in BBs. The following parts will describe how the BBs can be applied for use in defect detection on patterned fabric.

\subsection{The Formula of BBs}

The formula of BBs used for defect detection is defined as follows. For a particular row in an image of size $p x q$, the middle band (moving average) is defined as

$M_{r_{n}}=\frac{\left(\sum_{j=r_{1}}^{r_{n}} x_{j}\right)}{n}$,

where $n$ is an integer value denoting the length of period, and $x_{j}$ is the pixel value. The summation is from $r_{1}^{\prime}$ th pixel to $r_{n}^{\prime}$ th pixel with $1 \leq r_{1} \leq r_{n} \leq q$, where the upper band is defined as
$U_{r_{n}}=M_{r_{n}}+d \cdot \sigma_{r_{n}}$,

and the lower band is defined as

$L_{r_{n}}=M_{r_{n}}-d \cdot \sigma_{r_{n}}$,

where $d$ denotes the number of standard deviation, and $\sigma_{r_{n}}$ is defined as

$\sigma_{r_{n}}=\sqrt{\frac{\sum_{j=r_{1}}^{r_{n}}\left(x_{j}-M_{r_{n}}\right)^{2}}{n}}$.

The nominal values for $n$ and $d$ are 20 and 2, respectively. ${ }^{42}$

\subsection{Defect Detection Procedures on Patterned Fabric Using BBs}

For defect detection on patterned fabric, the application of BBs is divided into two stages: training and testing. The test dot-patterned jacquard fabric consists of a "repetitive unit," which is of size $15 \times 25$ pixels.

\subsubsection{Training stage}

Suppose there are $w$ reference histogram equalized images of size pxq.

Step 1. Apply BB on rows. For each row, use BB functions, Eqs. (1) to (4), to calculate the upper, middle, and lower bands for rows of every input image. Let $n=N 1$ be the length of the period [with respect to the length of the row in the repetitive unit in Fig. 5(b)] and $d=d 1$ be the number of standard deviation for BB on rows. An example of upper, middle, and lower BBs for a sample row from the first reference image is shown in Fig. 7. Furthermore, mesh diagrams (three-dimensional color diagram of matrix values shown by MATLAB) of lower bands and upper bands, on rows, of the first reference image $(N 1=40, d 1=2)$ are given in Figs. 8 and 9, respectively. These mesh diagrams show periodic waveforms for both lower and upper BBs.

(a) From Fig. 7, for the $k^{\prime}$ th row of the first reference image $R$, the upper limit of the upper band channel is $U^{\max , k}$ and the lower limit is $U^{\min , k}$. Then, for all $p$ rows in the first reference image, there will be $p$ upper limits of upper bands, namely as $\left\{U^{\max , 1}, U^{\max , 2}, \ldots, U^{\text {max }, p}\right\}$, and $p$ lower limits of lower bands in $p$ rows, namely as $\left\{U^{\min , 1}, U^{\min , 2}, \ldots, U^{\min , p}\right\}$.

(b) For the $R$ th reference image, the average of upper limits is defined as $U^{\text {avg } \max , R}=(1 / p) \sum_{i=1}^{p} U^{\max , i}$. The average of lower limits is defined as $U^{\text {avg } \min , R}=(1 / p) \sum_{i=1}^{p} U^{\mathrm{min}, i}$.

(c) The final average value of $w$ average upper limits of the upper band is $U_{\max }^{r o w}=(1 / w) \sum_{j=1}^{w} U^{a v g} \max , j$. The final average value of $w$ average lower limits of the upper band is $U_{\min }^{\text {row }}=(1 / w) \sum_{j=1}^{w} U^{a v g} \min , j$. Hence, one set of threshold values of the upper BB for rows is attained as $T_{\text {upper }}^{\text {row }}=\left\{U_{\max }^{\text {row }}, U_{\min }^{\text {row }}\right\}$.

(d) For the lower BB for rows, repeat the steps from (a) to (c) and another set of threshold values will be attained, $T_{\text {lower }}^{\text {row }}=\left\{L_{\max }^{\text {row }}, L_{\min }^{\text {row }}\right\}$. 


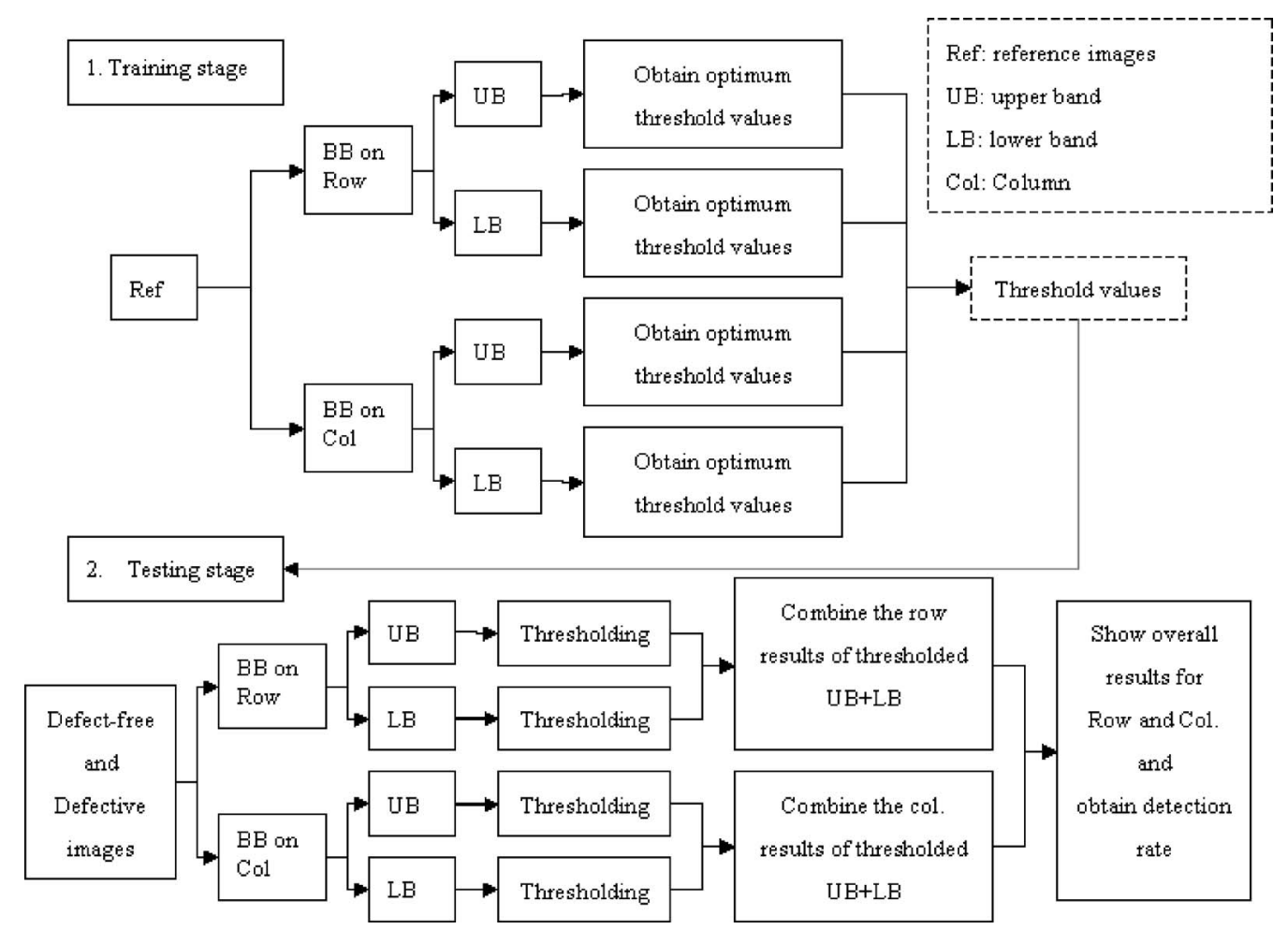

Fig. 12 The flowchart of BBs on defect detection.

Step 2. Repeat the same procedures in Step 1 for BB on columns. Let $n=N 2$ be the length of period and $d=d 2$ be the standard deviation for applying BB on columns. Two more sets of threshold values of upper and lower BBs for columns are attained, $T_{\text {upper }}^{\text {column }}=\left\{U_{\max }^{\text {column }}, U_{\min }^{\text {column }}\right\}$ and $T_{\text {lower }}^{\text {column }}=\left\{L_{\max }^{\text {column }}, L_{\min }^{\text {column }}\right\}$.

\subsubsection{Testing stage}

Suppose there is a histogram equalized test image [Fig. 6(e)] of size pxq.

Step 1. Apply $\mathrm{BB}$ on rows for the image. Using $\mathrm{BB}$ functions, Eqs. (1) to (4), to calculate the upper, middle, and lower bands of the input image. Two examples of mesh diagrams of lower bands and upper bands, on rows, of a defective fabric image with thin bar $(N 1=40, d 1=2)$ are given in Figs. 10 and 11, respectively. At some positions of the defective regions, there are substantial and obvious changes, especially from longitudinal views of diagram, Figs. 10(b) and 11(b). Then, we should threshold the upper and lower bands for each image by threshold values from the training stage and obtain $F_{U B}^{\text {row }}$ and $F_{L B}^{\text {row }}$, respectively. Lastly, we combine the thresholded results for upper and lower BBs for the test image, $F^{\text {row }}=F_{U B}^{\text {row }} \cup F_{L B}^{\text {row }}$.

Step 2. Apply BB on columns on the image. Repeat the procedures of Step 1.

Step 3. Combine the results of thresholded results of $\mathrm{BB}$ on rows and columns $F=F^{\text {row }} \cup F^{\text {column }}$. Determine whether the image is detected correctly.

\subsection{Discussion on Training for a Threshold}

The threshold values for upper and lower BBs are determined by training several reference images. For a reference image, hills and troughs would appear on the mesh diagrams for both upper and lower BBs in Figs. 8 and 9. For example, for $\mathrm{BBs}$ applied on rows, the threshold matrix $F_{\text {upperband }}^{\text {row }}$ on upper bands can be achieved by

$F_{\text {upperband }}^{\text {row }}=\left(f_{x, y}\right)= \begin{cases}1 & U(x, y)<U_{\min }^{\text {row }} \text { or } U(x, y)>U_{\max }^{\text {row }}, \\ 0 & \text { otherwise }\end{cases}$

where $T_{\text {upper }}^{\text {row }}=\left\{U_{\max }^{\text {row }}, U_{\min }^{\text {row }}\right\}$ is the threshold set for upper BB on rows, $U(x, y)$ is the value at $(x, y)$ position from the upper band matrix of the input image. A similar procedure is carried out for the lower bands. A defective image would show a subtle change after applying BB (e.g., Figs. 10 and 11). Because defective regions may not be tracked by thresholding on one particular band, there is a need to combine the thresholded results of both upper and lower bands when BB are applied on rows. Then, the same approach for $\mathrm{BB}$ on columns is undergone. Finally, the detection results are obtained from the combination of thresholded results from both rows and columns.

\section{Detailed Evaluation based on Dot-Patterned Fabric}

\subsection{Detection Success Rate on 230 Images}

A summary of the procedures on the application of BBs is presented in Fig. 12. A total of 230 samples (110 defect- 


\begin{tabular}{|c|c|c|c|c|}
\hline The statistical measures of 110 defect-free images & Mean & Max & Min & $\begin{array}{l}\text { Slandand } \\
\text { deviationt }\end{array}$ \\
\hline $\begin{array}{c}\text { No. of white pizels in final threshold images, } \\
\text { all in size of } 232 \times 242,(\mathrm{Nl}=15, \mathrm{dl}=2) \text { and }(\mathrm{N} 2=25, \mathrm{~d} 2=2)\end{array}$ & 11.6 & 31 & 1 & 6.57 \\
\hline
\end{tabular}

Fig. 13 Statistical detection results of 110 defect-free images.

free images and 120 defective images) from 10 types of defects of dot-patterned fabric have been tested in this section. Each input image is of size $256 \times 256$ pixels in gray level scale. The computer used for the evaluation in this paper is Pentium 4, 1.8 GHz, 256 DDR RAM. The computation speed for each input image is $27.8 \mathrm{~s}$ when implemented using MATLAB (version 6.5) and $0.47 \mathrm{~s}$ using VISUAL C\#. Hence, when implemented using the $C$ programming language, the developed method is fast enough for real-time on-loom defect detection.

For row detection from BB, the period length $(N)$ and standard deviation $(d)$ are 15 and 2, respectively. For column detection, the two values are 25 and 2 . The parameter $N$ depends on the nature of the texture of the patterned fabric that we have used in the evaluation. The values are obtained by the use of autocorrelation of the fabric texture. The size of a repetitive unit of the patterned fabric is then determined, which is the parameter $N$ in the paper. The other parameter $d$ is determined according to the original suggestion in the equation by John Bollinger. In Sec. 4.3 of this paper, the effect of varying the value of $d$ is also presented. It has been found that the value of 2 should be used as a nominal value of the method as in the original equation of the method.

For evaluation, a defective image is considered as one exceeding 50 white pixels out of $56144(0.089 \%$ of total pixels). The explanation follows. Two tables of statistical detection results are given in Figs. 13 and 14. In the training stage, there are two distinguished sets of white pixels emerging on the resultant images from the defect-free and defective images. Most resultant images from defect-free images emitted less than 30 white pixels. As shown in Fig. 13 at the testing stage for defect-free images, the mean is 11.6 white pixels, while that of defective images emitted over 100 white pixels. As shown in Fig. 14 at the testing stage for defective images, the minimum mean among all defect classes is 119.17 over 56144 pixels. If the range [ 0 , $30]$ is allocated for defect-free images and the range [50, $56144]$ is allocated for the defective images, then the range $[30,50]$ is the tolerance region for defect-free images. However, for a more strict criterion, the boundary is set at 50 . The number of white pixels after thresholding is used to determine whether an input fabric image is defective or not. The number of white pixels (50) is chosen as a heuristic value. It is based on a preliminary study of the BB method on the dot-patterned jacquard fabric. Then, this value is once again confirmed in Sec. 4.1 and the two tables of Figs. 13 and 14. The same heuristic value is simply used when carrying out the BB method for defect detection of starpatterned and box-patterned fabrics. It has been found that all 110 defect-free images and 119 out of 120 defective images can be correctly detected after thresholding. The detection success rate is $100 \%$ for defect-free images and $99.17 \%$ for defective images. Therefore, the overall detection success rate is $99.57 \%$. The result is very satisfactory and encouraging for defect detection on this kind of dotpatterned fabric. Some examples of those ten kinds of defective images (broken end, holes, knots, netting multiple, thick bar, thin bar, oil warp, oil weft, loose pick, and missing pick) are shown in Fig. 15.

\subsection{Effect Due to Variation of Period Length (N1, N2)}

Let $N 1$ and $N 2$ be the period lengths used in the row and column calculations of the $\mathrm{BB}$ equations. The number of standard deviation, $d 1$ and $d 2$, for $\mathrm{BB}$ on rows and columns have been fixed at 2 . As the repetitive unit of the dotpatterned jacquard fabric is of size $15 \times 25, N 1$ and $N 2$ have been set as 15 and 25, respectively. In order to investigate the variations of period length for $\mathrm{BB}$ in rows and

\begin{tabular}{|c|c|c|c|c|c|}
\hline \multirow{2}{*}{$\begin{array}{l}\text { Different kinds of } \\
\text { defective } \\
\text { Images }\end{array}$} & \multirow{12}{*}{$\begin{array}{l}\text { No. of white } \\
\text { pizels in final } \\
\text { threshold images, } \\
\text { all in size of } \\
232 \times 242\end{array}$} & \multicolumn{4}{|c|}{$\begin{array}{l}\text { The statistical measures of } 12 \text { ] defective images } \\
\text { Each kind of defects has } 12 \text { images }\end{array}$} \\
\hline & & Mean & Max & Min & Standard deriation \\
\hline Broken End & & 159.33 & 324 & 94 & 09.78 \\
\hline Hole & & 778.08 & 1226 & 381 & 270.96 \\
\hline Knots & & 940.92 & 1756 & 348 & 422.65 \\
\hline Netting Multiple & & 947.5 & 1765 & 454 & 398.04 \\
\hline Thin Bar & & 1625.83 & 3103 & 870 & 676.74 \\
\hline Thick Bar & & 5632.08 & 9539 & 2998 & 2286.80 \\
\hline Oil Warp & & 578.92 & 1131 & 187 & 280.93 \\
\hline Oil Weft & & 180.58 & 304 & 81 & 70.31 \\
\hline Laose Fick & & 119.17 & 2003 & 15 & 53.51 \\
\hline Missing Pick & & 324,42 & 925 & 84 & 224.26 \\
\hline
\end{tabular}

Fig. 14 Statistical detection results of 120 defective images. 


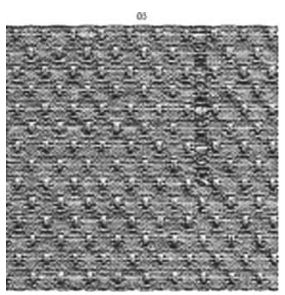

a. B token End( B )

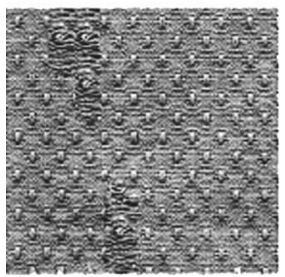

b. Holes ( $\mathrm{H}$ )

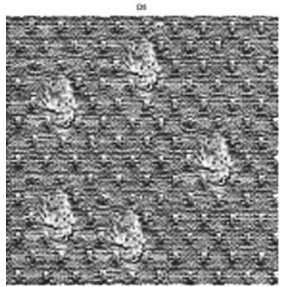

c. Knots (K)

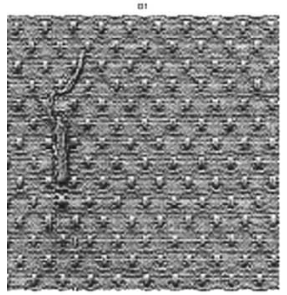

d. Netting Multiple ( N)

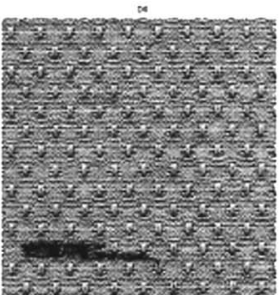

e. Thin $\operatorname{Bar}(\operatorname{Tn})$

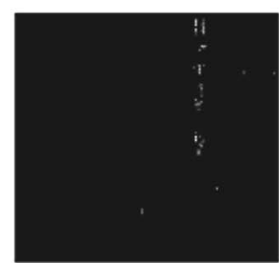

B

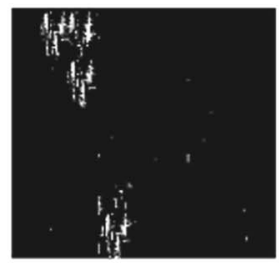

$\mathrm{H}$

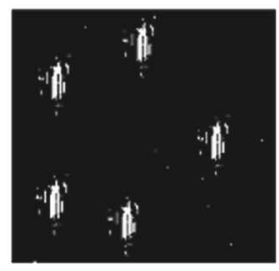

K

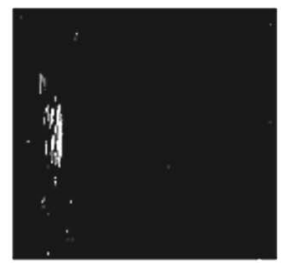

N

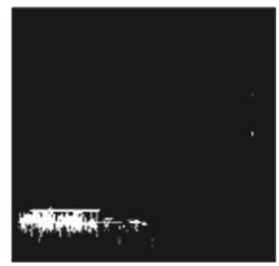

$\operatorname{Tn}$

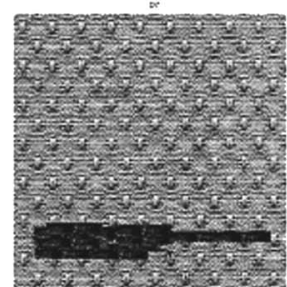

f. Thick Bar ( Tk)

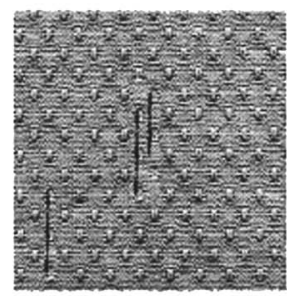

g. Oil Warp (Op)

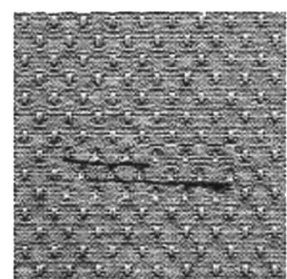

h. Oil Weft( Ot)

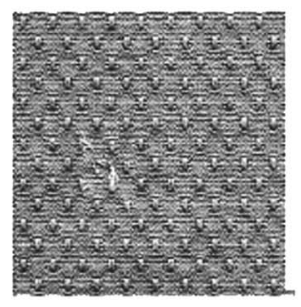

i. Loose Pick ( L )

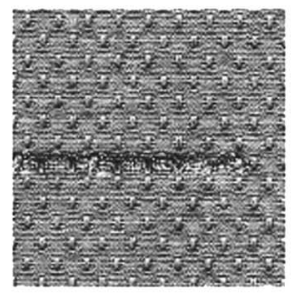

j. Missing Pick (M)

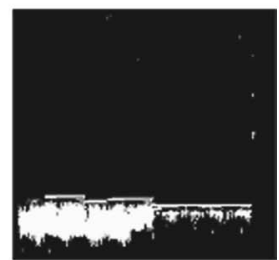

Tk

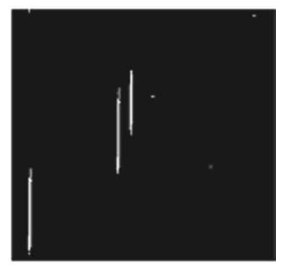

$\mathrm{Op}$

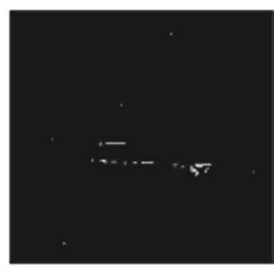

Ot

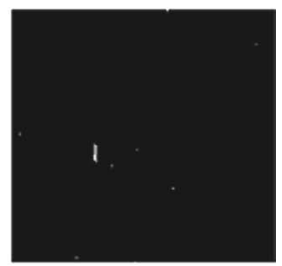

L

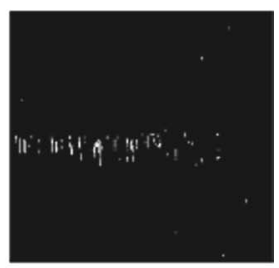

IVI

Fig. 15 Ten typical examples of the 10 kinds of defective images in testing set $(N 1=15, d 1=2$ and $N 2=25, d 2=2$ in $B B$ ).

columns, six other combinations of $N 1$ and $N 2$ are used. Figure 16 gives the detection result of the seven combinations of $N 1$ and $N 2$.

Detection results with $N 1 \geq 15$ and $N 2 \geq 25$ are similar to the combinations 3 to 7 . If $N 1$ and $N 2$ are set to be larger than one period length on rows and columns, the results are similar except for having denser outlined defective regions and more noises. If the values of $N 1$ and $N 2$ are chosen to be larger, the size of final thresholded image will be smaller when compared with smaller $N 1$ and $N 2$. Then, the defects near the border of the final thresholded image may be neglected. For combinations 1 and 2, less white pixels are detected in the selected defective samples of netting multiple. Figure 17 illustrated the final thresholded defective images from combinations 1,3 , and 7 . As a result, $N 1$ (for $\mathrm{BB}$ on rows) for 15 and $N 2$ (for $\mathrm{BB}$ on columns) for 25 are chosen as the standard sizes for $\mathrm{BB}$ corresponding to the dimension of the repetitive unit of the dot-patterned fabric. 
Ngan and Pang: Novel method for patterned fabric inspection...

\begin{tabular}{|c|c|c|c|c|c|c|c|}
\hline \multirow{3}{*}{ Combination } & \multirow[t]{2}{*}{ N1 } & \multirow{2}{*}{ N2 } & \multicolumn{2}{|c|}{ Defect-free samples } & \multicolumn{2}{|c|}{$\begin{array}{c}\text { Defective samples of } \\
\text { netting multiple }\end{array}$} & \multirow{2}{*}{$\begin{array}{l}\text { Size of } \\
\text { final innage }\end{array}$} \\
\hline & & & $15 t$ & 2nd & $1 s \mathrm{t}$ & 2nd & \\
\hline & $d 1=2$ & $d 2=2$ & \multicolumn{2}{|c|}{ No of white pixels } & \multicolumn{2}{|c|}{ No. of white pixels } & \\
\hline 1 & 5 & 15 & 5 & 2 & 229 & 526 & $242 \times 252$ \\
\hline 2 & 15 & 15 & 5 & 6 & 231 & 530 & $242 \times 242$ \\
\hline $3^{*}$ & 15 & 25 & 4 & 10 & 491 & 1052 & $232 \times 242$ \\
\hline 4 & 25 & 25 & 10 & 12 & 493 & 1064 & $232 \times 232$ \\
\hline 5 & 15 & 40 & 3 & 7 & 726 & 1504 & $242 \times 217$ \\
\hline 6 & 40 & 25 & 20 & 7 & 526 & 1057 & $217 \times 242$ \\
\hline 7 & 40 & 40 & 18 & 3 & 758 & 1460 & $217 \times 217$ \\
\hline
\end{tabular}

Fig. 16 Number of white pixels found in final threshold defect-free and defective images under variation of $N 1$ and $N 2$ for detection using BBs. Combination $3^{*}$ is the standard version of $N 1$ and $N 2$ used in the dot-patterned fabric defect detection in this dot-patterned fabric.

\subsection{Effect Due to Variation on the Number of Standard Deviation $(d 1, d 2)$}

In this investigation, $N 1$ and $N 2$ have been fixed at the standard combination 3 above. There are six combinations of $d 1$ and $d 2$ in this evaluation. First, by definitions of BB, the processes of applying $\mathrm{BB}$ on rows and columns are similar. For simplicity, from the combinations 1 to $4, d 1$ and $d 2$ are set to be equivalent and tuned from 1,2 , and 3

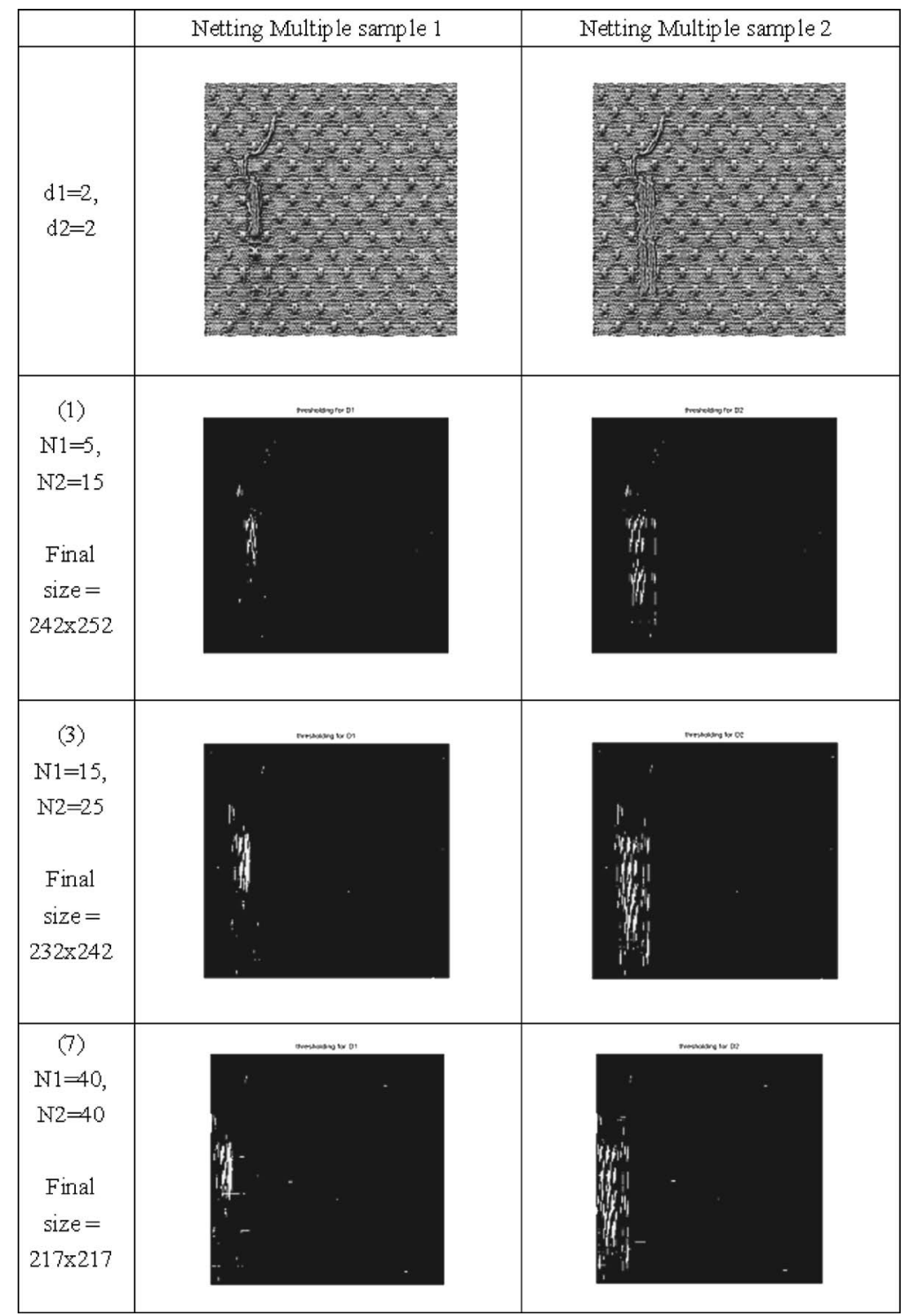

Fig. 17 Detection results of different combination of $N 1$ and $N 2$ by fixed $d 1$ and $d 2$. 
Ngan and Pang: Novel method for patterned fabric inspection...

\begin{tabular}{|c|c|c|c|c|c|}
\hline \multirow{2}{*}{ Combination } & \multirow{2}{*}{$\begin{array}{l}\text { Number of } \\
\text { standard } \\
\text { deviation } \\
\text { (d1 and } d 2)\end{array}$} & \multicolumn{2}{|c|}{ Defect-free samples } & \multicolumn{2}{|c|}{$\begin{array}{c}\text { Defective samples of } \\
\text { netting multiple }\end{array}$} \\
\hline & & $15 t$ & $2 n d$ & $1 s t$ & $2 \mathrm{nd}$ \\
\hline & $\mathrm{N} 1=15, \mathrm{~N} 2=25$ & \multicolumn{2}{|c|}{ No of white pixels } & \multicolumn{2}{|c|}{ No of white pixels } \\
\hline 1 & $d 1=d 2=1$ & 3 & 8 & 422 & 700 \\
\hline $2 *$ & $\mathrm{~d} 1=\mathrm{d} 2=2$ & 4 & 10 & 491 & 1052 \\
\hline 3 & $d 1=d 2=3$ & 6 & 8 & 479 & 1145 \\
\hline 4 & $\mathrm{~d} 1=\mathrm{d} 2=10$ & 12 & 10 & 382 & 1097 \\
\hline 5 & $d 1=1, d 2=10$ & 14 & 11 & 384 & 1094 \\
\hline 6 & $d 1=10, d 2=1$ & 1 & 7 & 424 & 703 \\
\hline
\end{tabular}

Fig. 18 Number of white pixels found in final threshold defect-free and defective images under variation of $d 1$ and $d 2$ for detection using BBs. $N 1$ and $N 2$ are fixed to be 15 and 25 . Combination $2^{*}$ is the standard version of $d 1$ and $d 2$ used in the dot-patterned fabric defect detection in this dot-patterned fabric.

to 10 . Then, the combinations 5 and 6 are used to test different allocations on the number of standard deviation. The results of detection are shown in Fig. 18. Three selected detection results for defective images with netting in multiple combinations 1, 5, and 6 are given in Fig. 19. The results are very similar for a different number of standard deviations. It coincides with the original definition on the use of the number of standard deviations in BBs by John

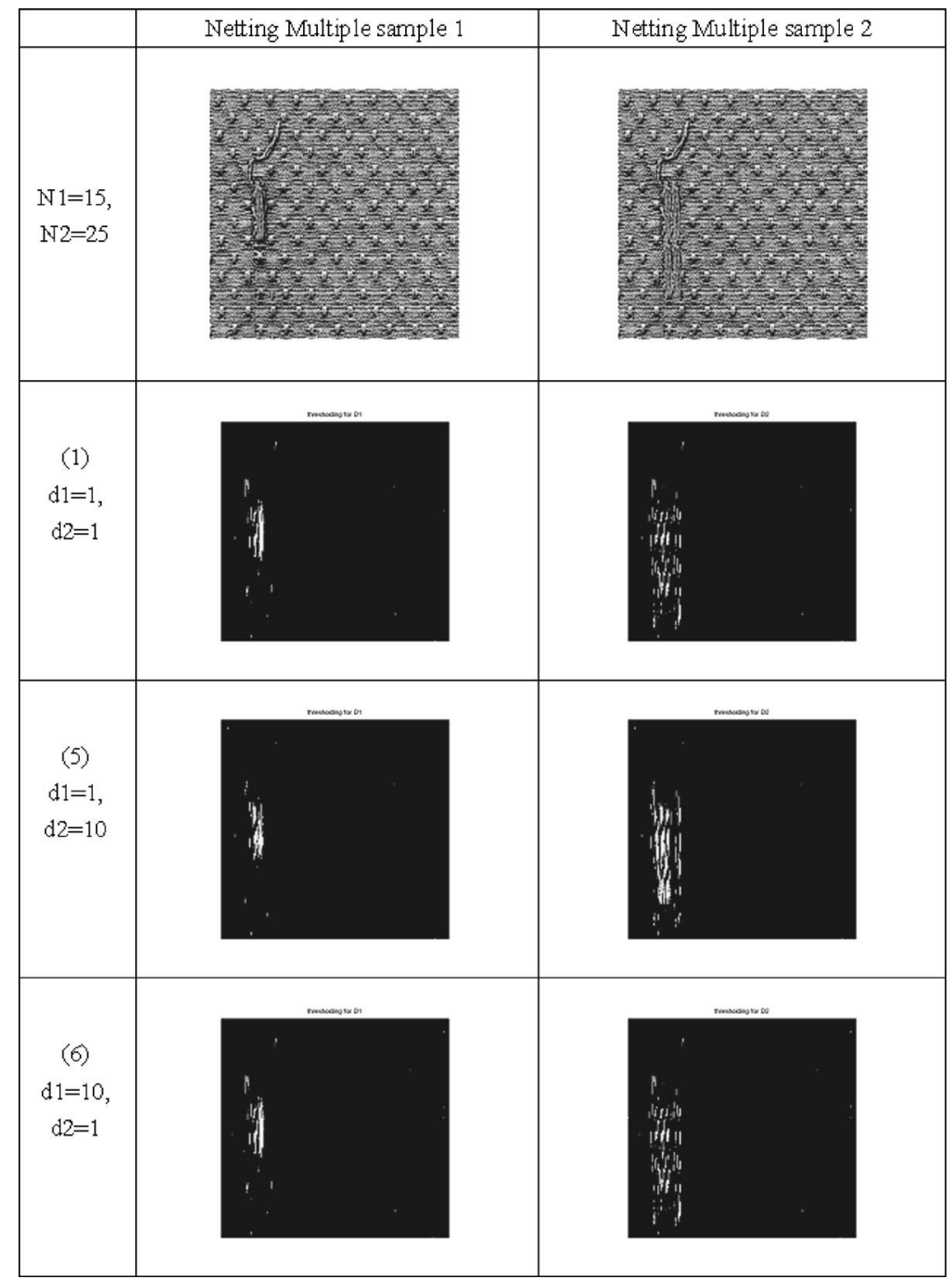

Fig. 19 Detection results of different combination of $d 1$ and $d 2$ by fixed $N 1$ and $N 2$. 


\begin{tabular}{|c|c|c|c|c|c|}
\hline \multicolumn{2}{|c|}{$\begin{array}{l}\text { Number of white pixels in final threshold images, } \\
\qquad(\mathrm{N} 1=\mathrm{N} 2=40, \mathrm{~d} 1=\mathrm{d} 2=2)\end{array}$} & Mean & $\operatorname{Max}$ & Min & $\begin{array}{l}\text { Standand } \\
\text { deviation }\end{array}$ \\
\hline \multicolumn{2}{|c|}{25 defect-free images } & 9.08 & 36 & 1 & 8.47 \\
\hline \multirow{5}{*}{25 defective images } & 5 Broken End & 375.2 & 1202 & 46 & 477.04 \\
\hline & 5 Hole & 103.6 & 132 & 59 & 29.79 \\
\hline & 5 Netting Multiple & 381.8 & 994 & 173 & 344.41 \\
\hline & 5 Thick Bar & 10456 & 3021 & 60 & 11757 \\
\hline & 5 Thin Bar & 1642 & 255 & 68 & 88.97 \\
\hline
\end{tabular}

Fig. 20 Statistical detection result of defect-free and defective images (total of 50 images) of starpatterned fabric.

Bollinger. $^{42}$ The number of standard deviations is designed for controlling the bandwidth, the distance between the upper and lower bands. Tuning the number of standard deviations is expected to shift the location of bands so that it will have little effect on the detection results. In this paper, the number of standard deviations for $d 1$ and $d 2$ of $\mathrm{BB}$ are set to two.

\section{More Results Based on Star-Patterned and Box-Patterned Fabric}

There is a need to evaluate the effectiveness and feasibility of the BB method on other kinds of patterned fabric. Fifty images of a star-patterned fabric (25 defect-free and 25 defective samples) and 56 images of a box-patterned fabric (30 defect-free and 26 defective samples) have been used in an extended evaluation. Five classes of defects were chosen as defective samples: broken end, holes, netting multiple, thick bar, and thin bar.

The size of a repetitive unit for both fabrics are 25 $\times 25$ pixels. In the evaluation, the period lengths, $N 1$ and $N 2$, are chosen as 40 (1.5 times of standard lengths) and standard deviations $d 1$ and $d 2$, are chosen as 2 for row and column detection of BB. In Sec. 4.2, the lengths of $N 1$ and $N 2$ were shown to make the BB method perform well when they exceeded the standard lengths of the rows or columns of that repetitive unit. However, if the lengths of $N 1$ and $N 2$ were larger than two times the standard dimension, the size of the resultant image would be downsized too much. Hence, there is an advantage to confining the lengths of $N 1$ and $N 2$ to within 1 to 2 times of standard size. $N 1$ and $N 2$ were deliberatively selected as 1.5 times the standard lengths in the star-patterned and box-patterned fabrics. This is just to show the generalization ability of the BB method in the extensive evaluation. The detection results have demonstrated that the method is robust. Now, the size of the resultant image after thresholding is $217 \times 217$, totaling 47089 pixels. For classification, a resultant image that ex- ceeds 50 white pixels out of $47089(0.106 \%)$ is considered to be a defective image. For the star-patterned fabric, all 25 defect-free images were correctly detected and 24 out of 25 defective images have been correctly detected (Fig. 20). The overall detection success rate is $98 \%$. For the boxpatterned fabric, all 30 defect-free images and 25 out of 26 defective images are correctly detected (Fig. 21). The overall detection success rate is $98.21 \%$. Five typical examples of five defect classes of star-patterned and box-patterned fabrics are shown in Fig. 22.

\section{Summary and Conclusions}

In this paper, a novel method of patterned fabric defect detection based on BBs has been developed. The BB method has achieved an average of $98.59 \%$ detection success rate on three kinds of patterned fabric. Figure 23 shows the ratios of correctly detected and detection success rates of three kinds of patterned fabric. The concept of the moving average of BB has taken advantage of the characteristic of patterned fabric that generates periodic signals on rows and columns. By setting the parameters in the standard form, the method has already shown an excellent result as shown in Secs. 4 and 5.

The method of BBs on defect detection for repetitive patterned fabric has been shown to be very robust and effective. The strength of BBs is that they can capture the periodicity of the patterned fabric. The BBs can effectively reveal any variation or abnormal change in patterns. One superior point of BBs, when compared with other defect detection methods, is its one-dimensional approach when carrying out computations on the patterned fabric. The lengths of period ( $N 1$ and $N 2$ ) of $\mathrm{BB}$ can be optimized easily if it is selected larger than the period of a repetitive unit. Besides, the two numbers of standard deviation $(d 1$ and $d 2$ ) do not seem to affect very much on the detection results. The noises have only happened when very large values of period length ( $N 1$ and $N 2$ ) are chosen. Next, the

\begin{tabular}{|c|c|c|c|c|c|}
\hline \multicolumn{2}{|c|}{$\begin{array}{l}\text { Number of white pixels in final threshold images, } \\
\qquad(\mathrm{N} 1=\mathrm{N} 2=40, \mathrm{~d} 1=\mathrm{d} 2=2)\end{array}$} & Mear & $\operatorname{Max}$ & Min & $\begin{array}{l}\text { Standard } \\
\text { deviation }\end{array}$ \\
\hline \multicolumn{2}{|c|}{30 defect-free images } & 18.5 & 46 & 4 & 11.21 \\
\hline \multirow{5}{*}{26 defective images } & 5 Broken End & 399 & 474 & 303 & 71.80 \\
\hline & 5 Hole & 80 & 122 & 36 & 33.29 \\
\hline & 5 Netting Multiple & 1462 & 275 & 75 & 76.98 \\
\hline & 5 Thick Bar & 1934 & 4502 & 189 & 1834.10 \\
\hline & 6 Thin Bar & 878 & 2699 & 213 & 919.30 \\
\hline
\end{tabular}

Fig. 21 Statistical detection result of defect-free and defective images (total of 56 images) of boxpatterned fabric. 


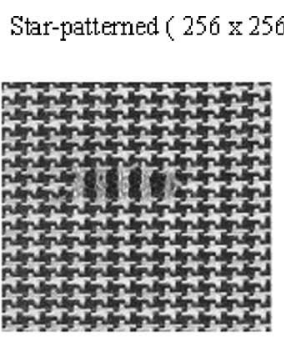

a. Broken End( $B$ )

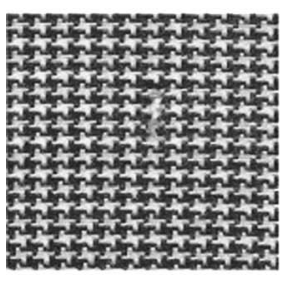

b. Holes (H)

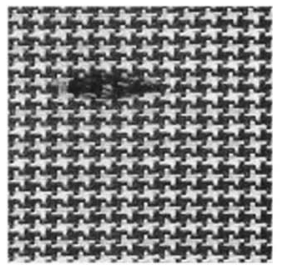

c. Netting Mul tiple (N)

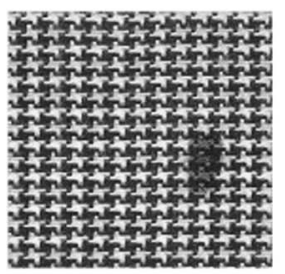

d. ThickBar ( Tk)

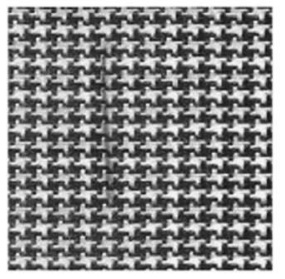

e. Thin Bar( $\mathrm{Tn})$
( $217 \times 217)$

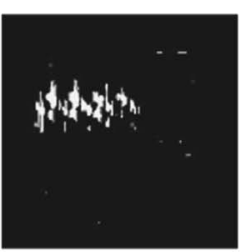

B

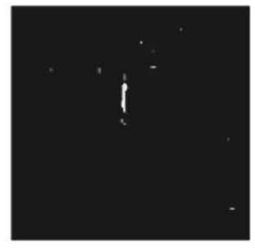

$\mathrm{H}$

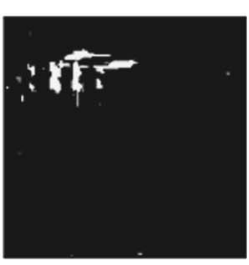

N

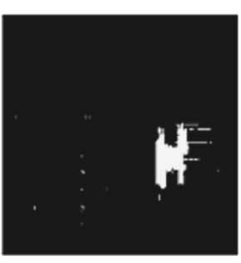

$\mathrm{Tk}$

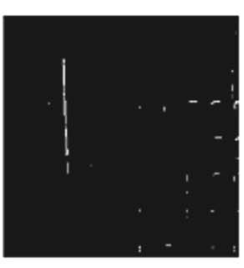

$\operatorname{Tn}$
Boz-patterned ( 256 × 256)

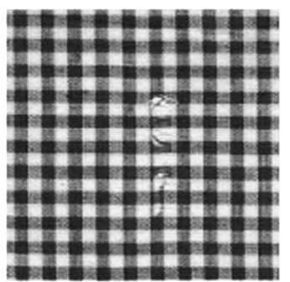

a. Broken End ( B )

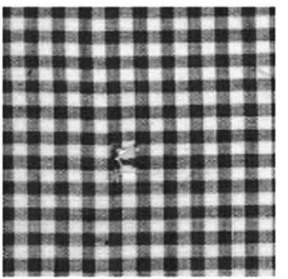

b. Holes ( $\mathrm{H}$ )

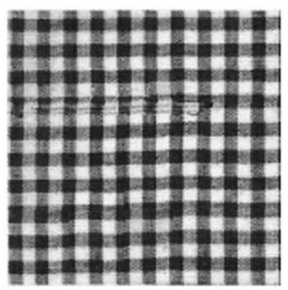

c. Netting Multiple (N)

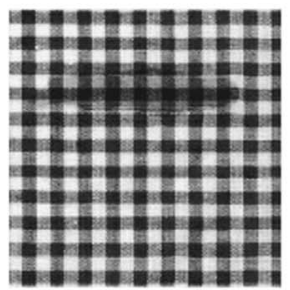

d. ThickBar ( Tk)

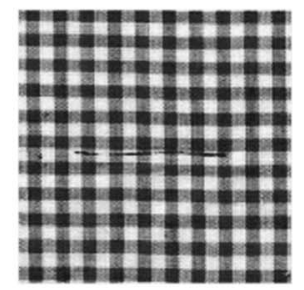

e. Thin Bar( $\operatorname{Tn})$
$(217 \times 217)$

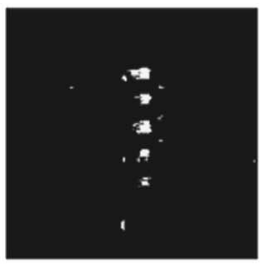

B

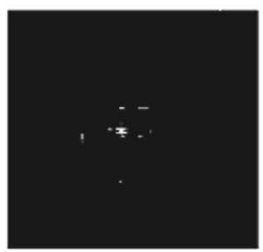

$\mathrm{H}$

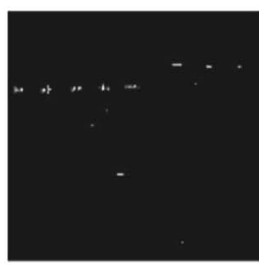

$N$

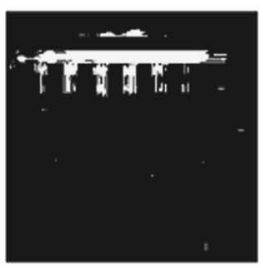

Tk

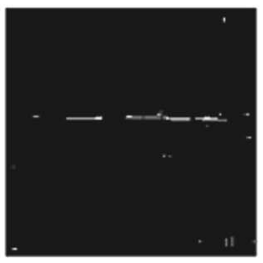

$\operatorname{Tn}$

Fig. 22 Five typical examples of five defect classes of star-patterned and box-patterned fabrics.

alignment problem between the reference image and test image in traditional subtraction can be avoided in the BB method, and it provides a practical advantage over the needs of fine tuning of scale and orientation of pixel-based comparison in Ref. 38 and the WGIS method in Ref. 41. Furthermore, the finalized detected image can be easily obtained and the thresholded images are crystal clean and clear without the needs of any postprocessing steps, such as the use of a smoothing filter to remove noises. $35,38,39,41$
Hence, the BB method outperforms the previous methods on patterned fabric inspection. Compared with other methods, the computation of the BB method is efficient and the detection algorithm can be easily implemented.

Some weaknesses of BBs should be mentioned. First, defects smaller than one repetitive unit may not be detected by the BB method. In this preliminary stage of application of the BB method, the detection is carried out with those defects usually larger than one repetitive unit on these three 
Ngan and Pang: Novel method for patterned fabric inspection...

\begin{tabular}{|c|c|c|c|}
\hline & $\begin{array}{l}\text { Ratio of correct detection } \\
\text { (Detection success rate of } \\
\text { defect-free images) }\end{array}$ & $\begin{array}{l}\text { Fatio of correct detection } \\
\text { (Detection success rate of } \\
\text { defective irrages) }\end{array}$ & $\begin{array}{l}\text { Ratio of correct detection } \\
\text { (Owerall detection } \\
\text { siccess rate) }\end{array}$ \\
\hline Dot-pattemed fabric & $110 / 110(100 \%)$ & $1101120(90.1 \%)$ & $2201230(00.57 \%)$ \\
\hline Star-patterned fahric & $25 / 25$ \{100\%? & $24125(96 \%)$ & $49 / 50(98 \%)$ \\
\hline B ox-frattemed fabric & $30 / 30(100 \%)$ & $25 / 26(96.15 \%)$ & $55 / 56(98.21 \%)$ \\
\hline \multicolumn{3}{|c|}{ Average arerall detection success rate } & $98.59 \%$ \\
\hline
\end{tabular}

Fig. 23 Ratios of correctly detected and detection success rates of three kinds of patterned fabric.

types of patterned fabric. The defective images that have not been detected as described in Fig. 23 are due to the small size of defects. Besides, those defects with only color differences from background, such as light-color-type oil stains, may not be detected because the method of BB is applied on the gray scaled images and not the RGB scaled images. It is most sensitive to the cases with big contrast between the defect region and background patterns. Lastly, a minor problem is that the borders of patterned fabric are neglected by definition of BB. However, it will not affect much because the period length of the pattern is relatively short compared to the width of fabric.

\section{Acknowledgment}

This research is support by a CRCG grant of the University of Hong Kong (CRCG Grant No. 10205131.23193.14300.323.01).

\section{References}

1. M. Bennamoun and A. Bodnarvova, "Automatic visual inspection and flaw detection in textile materials: Past, present and future," presented at Proc. IEEE SMC Conf., 4340-4343 (1998).

2. A. Bodnarova, M. Bennamoun, and K. K. Kubik, "Defect detection in textile materials based on aspects of HVS," presented at Proc. IEEE SMC Conf., San Diego, pp. 4423-4428 (1998).

3. C. H. Chan, H. Liu, T. Kwan, and G. Pang, "Automation technology for fabric inspection system," Proc. Conf. Appl. Automation Sci. Technol., City University of Hong Kong, pp. 24-26 (1998).

4. S. H. Hjimowlana, R. Muscedere, G. A. Jullien, and J. W. Roberts, "An in-camera data stream processing system for defect detection in web inspection tasks," Real-Time Imag. 5(1), 23-34 (1999).

5. L. H. Hoffer, F. Francini, B. Tiribilli, and G. Longobardi, "Neural networks for the optical recognition of defects in cloth," Opt. Eng. 35, 3138-3190 (1996).

6. I. S. Tsai, C. H. Lin, and J. J. Lin, "Applying an artificial neural network to pattern recognition in fabric defects," Text. Res. J. 65(3), $123-130(1995)$.

7. A. Kumar, "Neural network based detection of local textile defects," Pattern Recogn. 36, 1645-1659 (2002).

8. P. Mitropulos, C. Koulamas, R. Stojanovic, S. Koubias, G. Papadopoulos, and G. Karayanis, "Real-time vision system for defect detection and neural classification of web textile fabric," Proc. SPIE 3652, 59-69 (1999).

9. A. Tilocca, "Detecting fabric defects with a neural network using two kinds of optical patterns," Text. Res. J. 72(6), 545-551 (2002).

10. C. H. Chan and G. K. H. Pang, "Fabric defect detection by Fourier analysis," IEEE Trans. Ind. Appl. 36(5), (2000).

11. E. J. Wood, "Applying Fourier and associated transforms to pattern characterization in textiles," Text. Res. J. 60, 212-220 (1990).

12. C. Castellinin, F. Francini, G. Longobardi, and B. Tiribilli, "On-line textile quality control using optical Fourier transforms," Opt. Lasers Eng. 24, 19-32 (1996).

13. C. Cimberlini, F. Francini, G. Longobardi, P. Poggi, P. Sansoni, and B. Tiribilli, "Weaving defect detection by Fourier imaging," Proc. SPIE 2786, 9-18 (1996).

14. A. Kumar and G. K. H. Pang, "Defect detection in textured materials using Gabor filters," IEEE Trans. Ind. Appl. 38(2), 425-440 (2002).
15. A. Kumar and G. K. H. Pang, "Multi-channel defect segmentation using localized spatial filters," Opt. Eng. 39(12), 3176-3190 (2000).

16. A. Bodnarova, M. Bennamoun, and S. Lathm, "Optimal Gabor filters for textile flaw detection," Pattern Recogn. 35, 2973-2991 (2002).

17. J. Escofet, R. Navarro, M. S. Millan, and J. Pladellorens, "Detection of local defects in textile webs using Gabor filters," Opt. Eng. 37(8), 2297-2307 (1998).

18. L. H. Ding, L. Xiao, Y. W. Zhu, W. C. Liu, and Y. Liu, "Gabor filter based automatic textile defect detection," Proc. SPIE 4875, 789-795 (2002).

19. W. J. Jasper, S. J. Garnier, and H. Potlapalli, "Texture characterization and defect detection using adaptive wavelets," Opt. Eng. 35(11), 3140-3149 (1996)

20. M. Kreibl, H. Schwarzer, and S. Teiwes, "Optical processor for realtime detection of defects in textile webs," Proc. SPIE 3073, 307-311 (1997).

21. N. Blanchard, D. Prevost, and Y. Sheng, "Optical correlator for textile web defect detection," Proc. SPIE 4043, 280-290 (2000).

22. A. Latif-Amet, A. Ertuzun, and A. Ercil, "An efficient method for texture defect detection: Sub-band domain co-occurrence matrices," Image Vis. Comput. 18, 543-553 (1999).

23. H. Sari-Sarraf and J. S. Goddard, "Vision system for on-loom fabric inspection," IEEE Trans. Ind. Appl. 35(6), 1252-1259 (1999).

24. X. Yang, G. K. H. Pang, and N. Yung, "Fabric defect detection using adaptive wavelet," Proc. IEEE Conf. on Acoustic, Speech and Signal, 6(7-11), 3697-3700 (2001)

25. X. Yang, G. K. H. Pang, and N. Yung, "Discriminative fabric defect detection using adaptive wavelet," Opt. Eng., 41(12), 3116-3226 (2002).

26. J. L. Dorrity and G. Vachtsevanos, "On-line defect detection for weaving systems," presented at Proc. IEEE Tech. Conf. Text., Fiber, and Film Ind., pp. 1-6 (1996).

27. D. Chetverikov, "Structural defects: General approach and application to textile inspection," Proc. IEEE 15th Int. Conf. on Pattern Recog., 1, 521-524 (2000).

28. A. Bodmarova, M. Bennamoun, and K. K. Kubik, "Suitability analysis of techniques for flaw detection in textiles using texture analysis," Pattern Anal. Appl. 3(3), 254-266 (2000).

29. D. Puig and M. A. Garcia, "Pixel classification through divergencebased integration of texture methods with conflict resolution," Proc. IEEE Int. Conf. Image Process., 2, pp. II-1037-1040 (2003).

30. T. Li, P. Witty, and K. Tim, "Machine vision in the inspection on patterned textile webs," presented at IEE Colloquium on Industrial Inspection, Digest No. 1997/041, pp. 9/1-9/5, 10 February 1997.

31. C. Sandy, L. Norton-Wayne, and R. Harwood, "The automated inspection of lace using machine vision," Mechatronics 5(2/3), 215231 (1995).

32. R. T. Chin and C. A. Harlow, "Automated visual inspection: A survey," IEEE Trans. Pattern Anal. Mach. Intell. PAMI-4(6), 557-573 (1982).

33. M. F. M. Costa, F. Rodrigues, J. Guedes, and J. Lopes, "Automated evaluation of patterned fabrics for defect detection," Proc. SPIE 3831, 403-407 (2000).

34. T. Thomas and M. Cattoen, "Automatic inspection of simply patterned material in the textile industry," Proc. SPIE 2813, 2-12 (1994).

35. I. C. Baykal, R. Muscedere, and G. A. Jullien, "On the use of hash functions for defect detection in textures for in-camera web inspection systems," IEEE ISCAS 5, 665-668 (2002).

36. I. C. Baykal and G. A. Jullien, "Detection of defects in textures with alignment error for real-time line-scan web inspection systems," IEEE 45th MWSCAS, Vol. 3, pp. 292-295 (2002).

37. M. Bennamoun and A. Bodnarova, "Digital image processing techniques for automatic textile quality control," Syst. Anal. Model. Simul. 43(11), 1581-1614 (2003). 
38. U. Farooq, T. King, P. H. Gaskell, and N. Kapur, "Machine vision using image data feedback for fault detectionin complex deformable webs," Trans. Inst. Meas. Control (London) 26(2), 119-137 (2004).

39. H. Y. T. Ngan, G. K. H. Pang, S. P. Yung, and M. K. Ng, "Defect detection on patterned jacquard fabric," presented at IEEE Int. Workshop on 32nd Appl. Imagery Pattern Recogn., pp. 163-168 (2003).

40. C. Anagnostopoulos, I. Anagnostopoulos, D. Vergados, E. Kayafas, and V. Loumos, "Sliding windows: A software method suitable for real-time inspection of textile surfaces," Text. Res. J. 74(7), 646-651 (2004).

41. H. Y. T. Ngan, G. K. H. Pang, S. P. Yung, and M. K. Ng, "Wavelet based methods on patterned fabric defect detection," Pattern Recogn. 38, 559-576 (2005)

42. John Bollinger, Bollinger on Bollinger Bands, McGraw-Hill, New York (2001).

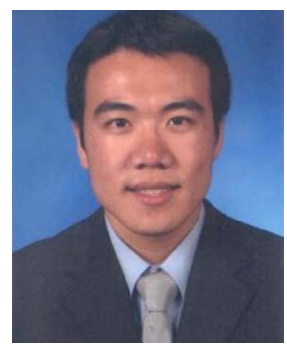

Henry Y. T. Ngan studied at The University of Hong Kong, HKSAR, China and received his BSc in mathematics in 2001 and his MPhil in electrical and electronic engineering in 2004. He is currently a PhD candidate and working in the Industrial Automated Research Laboratory of the Department of Electrical and Electronic Engineering at the University of Hong Kong. His current research topic is inspection of textured material and fabric, and his research interests include surface defect detection, image processing, pattern recognition, computer vision, and machine learning.

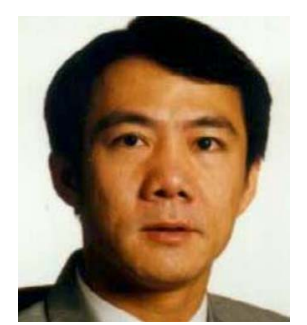

Grantham K. H. Pang obtained his PhD degree from the University of Cambridge in 1986. He was with the Department of Electrical and Computer Engineering, University of Waterloo, Canada, from 1986 to 1996 and joined the Department of Electrical and Electronic Engineering at the University of Hong Kong in 1996. Since 1988, he has published more than 130 technical papers and has authored or coauthored six books. $\mathrm{He}$ has also obtained five U.S. patents. His research interests include machine vision for surface defect detection, optical communications, expert systems for control system design, intelligent control, and intelligent transportation systems. He is in charge of the Industrial Automation Research Lab and has acted as a consultant to many companies, including Mitsubishi Electric Corp. in Japan, Northern Telecom and Imperial Oil Ltd. in Canada, MTR Corp. and COTCO Int. Ltd. in Hong Kong. In 1994, he worked as a senior visiting researcher at Hitachi Research Lab in Japan. He is a chartered electrical engineer and a member of IEE, HKIE as well as a senior member of IEEE. 